\title{
The traversable wormhole with classical scalar fields
}

\author{
Sung-Won Kim \\ Department of Science Education and \\ Center for High Energy Astrophysics and Isotope Studies(CAIS) \\ Ewha Womans University \\ Seoul 120-750, Korea \\ Sang Pyo Kim \\ Department of Physics \\ Kunsan National University \\ Kunsan 573-701, Korea
}

04.20.Gz,03.50.-z

*e-mail: sungwon@mm.ewha.ac.kr

†e-mail: sangkim@knusun1.kunsan.ac.kr 


\begin{abstract}
We study the Lorentzian static traversable wormholes coupled to quadratic scalar fields. We also obtain the solutions of the scalar fields and matters in the wormhole background and find that the minimal size of the wormhole should be quantized under the appropriate boundary conditions for the positive nonminimal massive scalar field.
\end{abstract}


One of the most important issues in making a practically usable Lorentzian wormhole is just the traversability [1,2]. To make a Lorentzian wormhole traversable, one has usually used an exotic matter which violates the well-known energy conditions [1]. For instance, a wormhole in the inflating cosmological model still requires the exotic matter to be traversable and to maintain its shape [3]. It is known that the vacuum energy of the inflating wormhole does not change the sign of the exoticity function. A traversable wormhole in the Friedmann-Robertson-Walker(FRW) cosmological model, however, does not necessarily require the exotic matter at the very early times 4 .

In this paper, we investigate the compatibility of a static wormhole with a minimal and a positive non-minimal scalar field. We also obtain the solutions to the matters and scalar fields in wormhole background. Furthermore, we find that the minimum size of wormhole should be quantized when appropriate boundary conditions are imposed for the non-minimal massive scalar field.

Firstly, we study the simplest case of a static Lorentzian wormhole with a minimal massless scalar field. The additional matter Lagrangian due to the scalar field is given by

$$
\mathcal{L}=\frac{1}{2} \sqrt{-g} g^{\mu \nu} \varphi_{; \mu} \varphi_{; \nu}
$$

and the equation of motion for $\varphi$ by

$$
\square \varphi=0 .
$$

The stress-energy tensor for $\varphi$ is obtained from Eq. (11) as

$$
T_{\mu \nu}^{(\varphi)}=\varphi_{; \mu} \varphi_{; \nu}-\frac{1}{2} g_{\mu \nu} g^{\rho \sigma} \varphi_{; \rho} \varphi_{; \sigma}
$$

Now the Einstein equation has an additional stress-energy tensor (3)

$$
G_{\mu \nu}=R_{\mu \nu}-\frac{1}{2} g_{\mu \nu} R=8 \pi T_{\mu \nu}=8 \pi\left(T_{\mu \nu}^{(\mathrm{w})}+T_{\mu \nu}^{(\varphi)}\right),
$$

where $T_{\mu \nu}^{(\mathrm{w})}$ is the stress-energy tensor of the background matter that makes the traversable wormhole. Assuming a spherically symmetric spacetime, one finds the components of $T_{\hat{\mu} \hat{\nu}}^{(\mathrm{w})}$ in orthonormal coordinates 


$$
T_{\hat{t} \hat{t}}^{(\mathrm{w})}=\rho(r, t), \quad T_{\hat{r} \hat{r}}^{(\mathrm{w})}=-\tau(r, t), \quad T_{\hat{\theta} \hat{\theta}}^{(\mathrm{w})}=P(r, t),
$$

where $\rho(r, t), \tau(r, t)$ and $P(r, t)$ are the mass energy density, radial tension per unit area, and lateral pressure, respectively, as measured by an observer at a fixed $r, \theta, \phi$.

The metric of the static wormhole is given by

$$
d s^{2}=-e^{2 \Lambda(r)} d t^{2}+\frac{d r^{2}}{1-b(r) / r}+r^{2}\left(d \theta^{2}+\sin ^{2} \theta d \phi^{2}\right) .
$$

The arbitrary functions $\Lambda(r)$ and $b(r)$ are lapse and wormhole shape functions, respectively. The shape of the wormhole is determined by $b(r)$. Beside the spherically symmetric and static spacetime, we further assume a zero-tidal-force as seen by stationary observer, $\Lambda(r)=$ 0 , to make the problem simpler. Thus not only the scalar field $\varphi$ but also the matter $\rho, \tau$, and $P$ are assumed to depend only on $r$. The components of $T_{\mu \nu}^{(\varphi)}$ in the static wormhole metric (6) have the form

$$
\begin{aligned}
T_{t t}^{(\varphi)} & =\frac{1}{2}\left(1-\frac{b}{r}\right) \varphi^{\prime 2} \\
T_{r r}^{(\varphi)} & =\frac{1}{2} \varphi^{\prime 2} \\
T_{\theta \theta}^{(\varphi)} & =-\frac{1}{2} r^{2}\left(1-\frac{b}{r}\right) \varphi^{\prime 2} \\
T_{\phi \phi}^{(\varphi)} & =-\frac{1}{2} r^{2}\left(1-\frac{b}{r}\right) \varphi^{\prime 2} \sin ^{2} \theta,
\end{aligned}
$$

where and hereafter a prime denoted the differentiation with respect to $r$. In the spacetime with the metric (6) and $\Lambda=0$, the field equation of $\varphi$ becomes

$$
\frac{\varphi^{\prime \prime}}{\varphi^{\prime}}+\frac{1}{2} \frac{(1-b / r)^{\prime}}{(1-b / r)}+\frac{2}{r}=0 \quad \text { or } \quad r^{4} \varphi^{\prime 2}\left(1-\frac{b}{r}\right)=\mathrm{const},
$$

and the Einstein equations are given explicitly by

$$
\begin{aligned}
\frac{b^{\prime}}{8 \pi r^{2}} & =\rho(r, t)+\frac{1}{2} \varphi^{\prime 2}\left(1-\frac{b}{r}\right), \\
\frac{b}{8 \pi r^{3}} & =\tau(r, t)-\frac{1}{2} \varphi^{\prime 2}\left(1-\frac{b}{r}\right), \\
\frac{b-b^{\prime} r}{16 \pi r^{3}} & =P(r, t)-\frac{1}{2} \varphi^{\prime 2}\left(1-\frac{b}{r}\right) .
\end{aligned}
$$


By redefining the effective matters by

$$
\begin{aligned}
& \rho_{\mathrm{eff}}=\rho+\frac{1}{2} \varphi^{\prime 2}\left(1-\frac{b}{r}\right), \\
& \tau_{\mathrm{eff}}=\tau-\frac{1}{2} \varphi^{\prime 2}\left(1-\frac{b}{r}\right), \\
& P_{\mathrm{eff}}=P-\frac{1}{2} \varphi^{\prime 2}\left(1-\frac{b}{r}\right),
\end{aligned}
$$

we are able to rewrite the Einstein equations as

$$
\begin{aligned}
\frac{b^{\prime}}{8 \pi r^{2}} & =\rho_{\mathrm{eff}}, \\
\frac{b}{8 \pi r^{3}} & =\tau_{\mathrm{eff}}, \\
\frac{b-b^{\prime} r}{16 \pi r^{3}} & =P_{\mathrm{eff}},
\end{aligned}
$$

Thus one sees that the conservation law of the effective stress-energy tensor $T_{\mu \nu}^{(\mathrm{w})}+T_{\mu \nu}^{(\varphi)}$ still obeys the same equation

$$
\tau_{\text {eff }}^{\prime}+\frac{2}{r}\left(\tau_{\text {eff }}+P_{\text {eff }}\right)=0
$$

We now find the solutions of scalar field and matter. To determine the spatial distributions of $b(r), \rho(r), \tau(r), P(r)$, and $\varphi(r)$, we need one more condition for them such as the equation of state, $P_{\text {eff }}=\beta \rho_{\text {eff }}$. With the appropriate asymptotic flatness imposed we find the effective matter as functions of $r$ [

$$
\begin{aligned}
\rho_{\mathrm{eff}}(r) & \propto r^{-2(1+3 \beta) /(1+2 \beta)}, \\
\tau_{\mathrm{eff}}(r) & \propto r^{-2(1+3 \beta) /(1+2 \beta)}, \\
b(r) & \propto r^{1 /(1+2 \beta)}, \quad \beta<-\frac{1}{2} .
\end{aligned}
$$

Since the scalar field effects changes just $r^{-4}$ by the field equation (11), the matter is given by

$$
\begin{aligned}
& \rho \sim r^{-2(1+3 \beta) /(1+2 \beta)}+r^{-4}, \\
& \tau \sim r^{-2(1+3 \beta) /(1+2 \beta)}+r^{-4} .
\end{aligned}
$$


Once $b(r)$ is known to depend on a specific value of $\beta$, we can integrate Eq. (11) to obtain

$$
\varphi(r) \propto \int \frac{d r}{r^{2} \sqrt{(1-b(r) / r)}} .
$$

For example, when $b=b_{0}^{2} / r$, where $\beta=-1$ and $b_{0}$ is the minimum size of the throat of the wormhole, we obtain that

$$
\varphi=\varphi_{0}\left[1-\arccos \left(\frac{b_{0}}{r}\right)\right]
$$

by assuming the boundary condition that $\varphi>0,\left.\varphi\right|_{r=b_{0}}=\varphi_{0}$, and $\lim _{r \rightarrow \infty} \varphi=0$. Thus the scalar field decreases monotonically, i.e. $\varphi^{\prime}<0$, and the matter has $\rho, \tau, P \propto r^{-4}$.

Secondly we consider a general quadratic scalar field, whose stress-energy tensor is given by

$$
\begin{aligned}
T_{\mu \nu}= & (1-2 \xi) \varphi_{; \mu} \varphi_{; \nu}+\left(2 \xi-\frac{1}{2}\right) g_{\mu \nu} \varphi_{; \alpha} \varphi^{; \alpha}-2 \xi \varphi \varphi_{; \mu \nu}+2 \xi g_{\mu \nu} \varphi \square \varphi \\
& +\xi\left(R_{\mu \nu}-\frac{1}{2} g_{\mu \nu} R\right) \varphi^{2}-\frac{1}{2} m^{2} g_{\mu \nu} \varphi^{2},
\end{aligned}
$$

where $\xi=0$ for minimal coupling and $\xi=\frac{1}{6}$ for conformal coupling. The mass of the field is given by $m$. The field equation for $\varphi$ is

$$
\left(\square-m^{2}-\xi R\right) \varphi=0
$$

For a non-minimal coupling there is a curvature effect of wormhole background to $T_{\mu \nu}$ and $\varphi$. The scalar curvature in the metric (6) with $\Lambda=0$ is given by

$$
R=\frac{2 b^{\prime}}{r^{2}}
$$

and the components of the stress-energy tensor for the scalar field by

$$
\begin{aligned}
T_{t t}^{(\varphi)}= & (1-4 \xi)\left[\frac{1}{2}\left(1-\frac{b}{r}\right) \varphi^{\prime 2}+\left(\frac{1}{2} m^{2}+\xi \frac{b^{\prime}}{r^{2}}\right) \varphi^{2}\right], \\
T_{r r}^{(\varphi)}= & \frac{1}{2} \varphi^{\prime 2}+\xi \frac{4}{r} \varphi^{\prime} \varphi-\left(1-\frac{b}{r}\right)^{-1}\left(\xi \frac{b}{r^{3}}+\frac{1}{2} m^{2}\right) \varphi^{2}, \\
T_{\theta \theta}^{(\varphi)}= & \left(1-\frac{b}{r}\right)\left[\left(2 \xi-\frac{1}{2}\right) r^{2} \varphi^{\prime 2}-2 \xi r \varphi^{\prime} \varphi\right] \\
& +\left[2 \xi r^{2}\left(m^{2}+\xi \frac{2 b^{\prime}}{r^{2}}\right)+\xi\left(\frac{b}{2 r}-\frac{b^{\prime}}{2}\right)-\frac{1}{2} m^{2} r^{2}\right] \varphi^{2}, \\
T_{\phi \phi}^{(\varphi)}= & T_{\theta \theta}^{(\varphi)} \sin ^{2} \theta .
\end{aligned}
$$


As in the minimally-coupled case, we are also able to find the redefinition of the effective matter

$$
\begin{aligned}
\rho_{\mathrm{eff}}= & \rho+(1-4 \xi)\left[\frac{1}{2}\left(1-\frac{b}{r}\right) \varphi^{\prime 2}+\left(\frac{1}{2} m^{2}+\xi \frac{b^{\prime}}{r^{2}}\right) \varphi^{2}\right], \\
\tau_{\mathrm{eff}}= & \tau-\frac{1}{2} \varphi^{\prime 2}\left(1-\frac{b}{r}\right)-\xi \frac{4}{r}\left(1-\frac{b}{r}\right) \varphi^{\prime} \varphi+\left(\xi \frac{b}{r^{3}}+\frac{1}{2} m^{2}\right) \varphi^{2}, \\
P_{\mathrm{eff}}= & P+\left(1-\frac{b}{r}\right)\left[\left(2 \xi-\frac{1}{2}\right) \varphi^{\prime 2}-\xi \frac{2}{r} \varphi^{\prime} \varphi\right] \\
& +\left[2 \xi\left(m^{2}+\xi \frac{2 b^{\prime}}{r^{2}}\right)+\xi\left(\frac{b}{2 r^{3}}-\frac{b^{\prime}}{2 r^{2}}\right)-\frac{1}{2} m^{2}\right] \varphi^{2} .
\end{aligned}
$$

One has the same solution to the equation of the effective matter as Eqs.(22-24) with the same equation of state. However, one has a more complicated scalar field equation

$$
\left(1-\frac{b}{r}\right) \varphi^{\prime \prime}+\left(1-\frac{b}{r}\right)^{\prime} \varphi^{\prime}+\frac{2}{r}\left(1-\frac{b}{r}\right) \varphi^{\prime}=\left(m^{2}+2 \xi \frac{b^{\prime}}{r^{2}}\right) \varphi
$$

¿From the wormhole shape function $b \sim r^{1 /(1+2 \beta)}$ with the same equation of state $P_{\text {eff }}=\beta \rho_{\text {eff }}$, the asymptotic form of the field can be calculated near the throat and at the infinity.

$$
\begin{aligned}
\varphi & \sim \frac{\exp \left[k m^{2} r^{2(1+3 \beta) /(1+2 \beta)}\right]}{r^{-2 \xi / \beta}} \sim \frac{\exp \left(k m^{2} / \rho_{\mathrm{eff}}\right)}{r^{-2 \xi / \beta}} \quad \text { at } r \rightarrow b \\
& \sim e^{-m r} \quad \text { at } r \rightarrow \infty,
\end{aligned}
$$

where $k=(1+2 \beta)^{2} /[2 \beta(1+3 \beta)] b_{0}^{-2 \beta /(1+2 \beta)}$. For the special case of $\beta=-1$, i.e., $b=b_{0}^{2} / r$, the scalar field has the asymptotic form near the throat

$$
\varphi \sim \frac{\exp \left(k m^{2} r^{4}\right)}{r^{2 \xi}}, \quad \text { at } r \rightarrow b
$$

where $k \rightarrow 1 / 4 b_{0}$. The scalar field begins to increase from the throat very rapidly with $r$, but decreases exponentially at the infinity.

To find the exact solution to the scalar field, we rewrite the field equation (39) as

$$
\frac{d^{2} \varphi}{d s^{2}}-r^{4}\left(m^{2}+\xi R\right) \varphi=\frac{d^{2} \varphi}{d s^{2}}-f(s) \varphi=0,
$$

where $s=\int r^{-2}(1-b / r)^{-1 / 2} d r$ and $f(s)=r^{2}(s)\left(m^{2}+2 \xi b^{\prime}(s) / r^{2}(s)\right)$. One may interpret Eq. (42) as a Schrödinger equation with the zero energy, which is, however, not easy to solve 
in general. When $m=\xi=0$, the field equation becomes just the minimal massless case and the solution is $\varphi \sim s$, which is given by Eq. (27). For the general case, we can make use of the analogy with a bounded potential problem in the region $r_{0} \leq r<\infty$ or $0 \leq s \leq s_{0}$, where $r_{0}$ is the place of the throat. In this region, we can find the asymptotic values of potential $f(s)$ and $\varphi$ as

$$
\begin{array}{rl}
\lim _{r \rightarrow r_{0} \text { or } s \rightarrow 0} f(s)<0, & \quad \lim _{r \rightarrow r_{0} \text { or } s \rightarrow 0} \varphi \sim 0, \\
\lim _{r \rightarrow \infty} \text { or } s \rightarrow s_{0} & f(s)=\infty, \quad \lim _{r \rightarrow \infty \text { or } s \rightarrow s_{0}} \varphi \sim 0,
\end{array}
$$

if $m^{2}+2 \xi b^{\prime}(s) / r^{2}<0$ near throat or $m^{2} r_{0}^{2}+2 \xi b^{\prime}(0)<0$. Otherwise, there is no solution to $\varphi$. The bounded potential shows that one parameter should be quantized. Which parameter is quantized?

We consider the specific case of $b=b_{0}^{2} / r$ as above. Then the field equation becomes

$$
\frac{d^{2} \varphi}{d s^{2}}+\left[2 \xi b_{0}^{2}-m^{2} b_{0}^{2} \sec ^{4}\left(b_{0} s\right)\right] \varphi=0
$$

where $s=\left(1 / b_{0}\right) \arccos \left(b_{0} / r\right)$. First, the massless scalar field has the solution

$$
\varphi=\varphi_{0} \cos \left(\sqrt{2 \xi} b_{0} s\right)=\varphi_{0} \cos \left[\sqrt{2 \xi} \arccos \left(\frac{b_{0}}{r}\right)\right] .
$$

Second, in the massive case, however, one has the energy parameter $E=2 \xi b_{0}^{2}$ and the potential $V=m^{2} b_{0}^{2} \sec ^{4}\left(b_{0} s\right)$. The relation $E>V$ or $2 \xi>m^{2} b_{0}^{2}$ must be satisfied to guarantee a bounded solution. For small $s$, that is the region near throat, Eq. (45) is approximately the harmonic oscillator problem

$$
\frac{d^{2} \varphi}{d x^{2}}+\left(\lambda-x^{2}\right) \varphi=0
$$

with $x^{2}=2 m b_{0}^{3} s^{2}$ and $\lambda=\left(2 \xi-m^{2} b_{0}^{2}\right) /\left(2 m b_{0}\right)$. The solution is the harmonic wave functions $\varphi_{n}=e^{-x^{2} / 2} H_{n}(x)$, the Hermite polynomial. ¿From the energy quantization, we get $m b_{0}=$ $2 n+1+\sqrt{(2 n+1)^{2}+2 \xi}$, where $n$ is odd number. ¿From this result we may conclude that $\xi b_{0}^{2}$ or the minimal size of the wormhole should be quantized for the non-minimal positive coupling $\xi>0$, including the conformal coupling $\xi=1 / 6$. 
In this paper we found the solutions of the wormhole with minimal and non-minimal scalar fields. For a positive non-minimal massive scalar field case we find that the size of throat, the minimal size of the wormhole, should be quantized in order to have the scalar

field solution satisfying the appropriate boundary conditions. We also find the solutions to the matter and the scalar field in each cases.

\section{ACKNOWLEDGMENTS}

This work was supported in part by Non-directed program of Ministry of Education, 1994, in part by the Basic Science Research Institute in Ewha Womans University, BSRI97-2427, and in part by the Korea Science and Engineering Foundation No. 95-0702-04-01-3. 


\section{REFERENCES}

[1] M. S. Morris, K. S. Thorne, Am. J. Phys. 56, 395 (1988).

[2] M. S. Morris, K. S. Thorne, U. Yurtsever, Phys. Rev. Lett. 61, 1446 (1988).

[3] T. Roman, Phys. Rev. D 47, 1370 (1993).

[4] Sung-Won Kim, Phy. Rev. D 53, 6889 (1996). 